\title{
\%
}

Pascual Palomares Miralles*

Joan Ripoll Alcón ${ }^{* *}$

\section{EL PAPEL DE LOS INCENTIVOS FISCALES A LA INVERSIÓN EN I+D+i EN ESPAÑA}

El propósito de este estudio es analizar el papel de los incentivos fiscales a la inversión en investigación, desarrollo e innovación (I+D+i) en España, y en particular la relevancia de la figura legal Patent Box basada en la cesión de uso de activos intangibles.

Este artículo ilustra, a partir de un análisis comparativo, cómo el esquema Patent Box supone un ahorro fiscal evidente respecto de aquella situación en la que no se hace uso del mismo. Este ahorro acaba erigiéndose en un incentivo fiscal efectivo en la promoción de proyectos de innovación empresarial, a diferencia de las deducciones fiscales, cuyo proceloso procedimiento administrativo y la fijación de un límite máximo de reducción de la cuota íntegra del impuesto de sociedades, que se ha revelado insuficiente, han tendido a minar su protagonismo como instrumento de ayuda indirecta a la financiación de la $\mathrm{I}+\mathrm{D}+\mathrm{i}$.

Palabras clave: incentivo fiscal, CDTI, Patent Box.

Clasificación JEL: L53, O31, O32, O38.

\section{Introducción}

Las teorías del crecimiento económico, tanto las que focalizan en factores exógenos (Solow, 1956) como en factores endógenos (Romer, 1994; Lucas, 1998), coinciden en que el aumento de la producción a largo plazo, o crecimiento potencial de una economía, se sustenta en el progreso técnico. De hecho, solo la mejora sostenida del progreso técnico evita el estancamiento de la actividad a largo plazo (estado estacionario) y garantiza un

* CEU Escuela Internacional de Doctorado (CEINDO).

** Departamento de Empresa y Economía. Universidad Abat Oliba CEU. Versión de junio de 2020

DOI: https:/doi.org/10.32796/bice.2020.3129.7115 crecimiento económico continuado a lo largo del tiempo.

Esta mejora del progreso técnico está estrechamente vinculada a innovaciones de proceso o de producto, determinantes para la productividad empresarial. A su vez, las innovaciones técnicas se vinculan directamente con las actividades de investigación y la transferencia tecnológica fundamental para el desarrollo económico. Por ello, la I+D+i constituye un elemento fundamental a la hora de explicar la evolución de la productividad y el crecimiento a largo plazo.

El artículo se estructura en tres partes. En la primera se definen los conceptos de investigación, desarrollo e innovación que enmarcan $\triangleright$ 
el análisis. La segunda parte describe las ayudas directas e indirectas que facilitan la financiación de las actividades de I+D+i, enfatizando la relevancia de la cesión de activos intangibles o Patent Box como incentivos fiscales a la innovación. Finalmente, el tercer apartado muestra las principales conclusiones del estudio realizado.

\section{2. ¿Qué se entiende por investigación, desarrollo e innovación?}

No existe una definición unificada para los conceptos de investigación, desarrollo e innovación, de manera que estas definiciones pueden variar en algunos matices en función del organismo que las utilice. Sin embargo, en nuestro análisis tomamos como referencia las definiciones establecidas en el Manual de Oslo ${ }^{1}$ de la OCDE y Eurostat (2018), puesto que son las que toma como base fundamental la legislación española sobre incentivos a la innovación.

En este sentido, entendemos como investigación (I) aquellas actividades cuya finalidad es la adquisición de nuevos conocimientos que puedan resultar de utilidad para la creación de nuevos productos, procesos o servicios o contribuir a mejorar considerablemente los ya existentes. De la misma manera, el desarrollo (D) se refiere a la adquisición, combinación, configuración y empleo de conocimientos y técnicas ya existentes, de índole científica, tecnológica, empresarial o de otro tipo, con vistas a la elaboración de planes y estructuras o diseños de productos, procesos o servicios nuevos,

El Manual de Oslo es la principal guía internacional para la realización de mediciones y estudios de actividades científicas y tecnológicas. Define conceptos de investigación y desarrollo y clarifica las actividades consideradas como innovadoras. modificados o mejorados. Se incluye la elaboración de proyectos, diseños, planos y demás tipos de documentación siempre y cuando no vaya destinada a usos comerciales, así como el desarrollo de prototipos y proyectos piloto. El desarrollo experimental no incluye las modificaciones habituales o periódicas efectuadas en los productos, líneas de producción, procesos de fabricación, servicios existentes y otras actividades en curso, aun cuando dichas modificaciones puedan representar mejoras.

Finalmente, se considera innovación (i) la aplicación de un método de producción o suministro nuevo o significativamente mejorado, incluyendo cambios significativos en cuanto a técnicas, equipos y/o programas informáticos, aplicación de nuevos métodos organizativos a las prácticas comerciales, la organización del centro de trabajo o las relaciones exteriores de la empresa, etc.

\section{La financiación de actividades de investigación, desarrollo, e innovación}

\subsection{Consideraciones previas}

La realización de actividades y proyectos de investigación, desarrollo e innovación ( $1+D+i)$ es un proceso arriesgado pero necesario para asegurar la competitividad de una empresa.

Las Administraciones públicas apoyan a las entidades que emprenden este tipo de actividades mediante dos mecanismos fundamentales: a) ayudas directas (como subvenciones, préstamos, ayudas o anticipos reembolsables ${ }^{2}$ y capital riesgo) y b) ayudas indirectas, esto es, $\square$

2 Las ayudas parcialmente reembolsables son préstamos a medio y largo plazo a tipo de interés fijo o variable por debajo de mercado con una parte que no debe devolverse. 
deducciones fiscales. Tanto en un caso como en otro, existe una premisa fundamental que establece que un proyecto de $\mathrm{I}+\mathrm{D}+\mathrm{i}$ no puede ser financiado con ayudas públicas en una cantidad mayor al coste total del proyecto, fijándose un límite máximo del $75 \%$ de su presupuesto.

Por otro lado, la intensidad máxima ${ }^{3}$ de las ayudas públicas está limitada de acuerdo con el riesgo técnico asumido por las empresas en la realización de las actividades de I+D+i. Con carácter general, las ayudas públicas a la realización de proyectos de investigación (lejanos al mercado) son mayores que las aportadas a proyectos de innovación (cercanos al mercado). Asimismo, la ejecución de proyectos en colaboración entre empresas o con organismos de investigación implica un incremento de la intensidad máxima de la ayuda que se puede conceder a un determinado proyecto.

\subsection{Ayudas de financiación directa}

\subsubsection{Esquemas de financiación directa}

Por lo general, para los proyectos de $\mathrm{I}+\mathrm{D}+\mathrm{i}$ existen dos modalidades fundamentales de ayudas a la financiación directa: subvenciones y préstamos. El esquema de financiación de cada proyecto dependerá de las características del mismo, de su nivel de riesgo y, en ocasiones, del tipo de empresa, existiendo la posibilidad de que un mismo proyecto reciba financiación combinando subvenciones y préstamos.

Otro esquema de financiación posible es el de la ayuda parcialmente reembolsable (APR), que consiste en un préstamo dividido en un tramo reembolsable y otro no reembolsable, que

3 La limitación de la intensidad máxima de ayudas se encuentra reflejada en el Marco sobre Ayudas Estatales de Investigación y Desarrollo e Innovación (2014/C 198/01). puede alcanzar hasta el $33 \%$ de la ayuda total, en función de las características del proyecto o el tamaño de la empresa.

\subsubsection{Organismos y niveles de financiación}

Las ayudas de financiación directa de las actividades de $\mathrm{I}+\mathrm{D}+\mathrm{i}$ son gestionadas por diversos organismos a distintos niveles. A nivel nacional, existen varios organismos que con sus programas contribuyen a la financiación de proyectos de $\mathrm{I}+\mathrm{D}+\mathrm{i}$ (además de las Cámaras de Comercio en el marco del programa Innocámaras): el Ministerio de Ciencia e Innovación y el Ministerio de Industria, Comercio y Turismo.

El Ministerio de Ciencia e Innovación, a través del Centro para el Desarrollo Tecnológico Industrial (CDTI), ofrece financiación para proyectos de investigación, desarrollo e innovación realizados por empresas, de manera individual o en cooperación. Sus ayudas, en los programas nacionales, no se rigen por convocatorias, lo que hace que se pueda presentar una solicitud de ayuda para un proyecto en cualquier momento. En cambio, los proyectos internacionales sí están sujetos a determinados plazos, que son publicados con antelación.

En el caso del Ministerio de Industria, Comercio y Turismo, a través de la Secretaría General de Industria y de la Pequeña y Mediana Empresa, con el ánimo de estimular la competitividad y mejorar la posición en el mercado de las empresas, se ha aprobado un marco normativo para la financiación de proyectos de investigación industrial, desarrollo experimental e innovación en materia de organización y procesos en el ámbito de la industria manufacturera. En particular, esta actuación de política industrial pretende apoyar el impulso tecnológico a empresas y sectores industriales con gran $\triangleright$ 
efecto tractor sobre otras actividades auxiliares y complementarias, así como a las cadenas de valor estratégicas.

A nivel regional, en las comunidades autónomas existen organismos que pueden ofrecer ayudas para la financiación directa de las actividades de I+D+i. Hoy en día, prácticamente la totalidad de las comunidades cuentan con planes de ciencia y tecnología, y sus líneas de apoyo a proyectos empresariales generalmente financian tanto actividades de I+D como de innovación. Habitualmente, los organismos gestores de las ayudas en el ámbito autonómico son las Consejerías de Industria, así como las Agencias de Desarrollo.

La Base de Datos de Ayudas e Incentivos para empresas recoge todas las medidas en forma de subvenciones, créditos e incentivos fiscales o avales, entre otros. Se trata de una iniciativa de la Dirección General de Industria y de la Pequeña y Mediana Empresa que recoge el esfuerzo de las Administraciones públicas y sus organismos para incentivar, mantener y desarrollar la actividad económica y el emprendimiento. Se alimenta de las convocatorias de los diarios oficiales: europeo, estatal, autonómicos, provinciales y locales; a los que se suma información de organismos oficiales que publicitan por otros medios.

\subsubsection{El ciclo de vida de una ayuda}

El ciclo de vida de un proyecto financiado en parte con fondos públicos consta de tres etapas fundamentales: solicitud, evaluación y desarrollo, y justificación.

\section{Solicitud de financiación}

En esta etapa se procederá a presentar el proyecto a la convocatoria pública seleccionada.
Cada convocatoria tiene sus características particulares, por lo que resulta importante conocer sus detalles. En términos generales, una solicitud de ayuda para un proyecto de I+D+i constará de los siguientes documentos: formulario de solicitud, memoria del proyecto, documentación administrativa y presupuesto del proyecto.

Esta etapa finaliza con la presentación formal de la solicitud al organismo correspondiente.

\section{Evaluación y desarrollo}

El organismo correspondiente evalúa la documentación presentada desde los puntos de vista formal, técnico y económico.

Como resultado de la evaluación, el organismo correspondiente procederá a comunicar al solicitante la propuesta de concesión (o denegación) del proyecto, indicando el presupuesto admitido como financiable o elegible del total del presupuesto presentado, así como el plazo para su ejecución.

\section{Justificación}

La justificación de la realización del proyecto es muy importante en lo que se refiere al cobro efectivo de la financiación concedida. La justificación implica dos actividades: 1) justificación técnica y 2) justificación económica.

La justificación técnica se refiere a la presentación de evidencias que constaten la realización de las actividades propuestas: cumplimiento de objetivos, planificación, resultados alcanzados, etc.

La justificación económica recoge la ejecución efectiva de los gastos previstos. Esto supone la presentación de justificantes de gastos y cualquier otra documentación adicional que nos pueda requerir el organismo gestor de la ayuda. 
En algunos casos, la justificación documental se acompaña de una visita de un técnico especialista, que comprueba la ejecución técnica del proyecto y la materialización de los gastos que realizase por parte de un organismo de control autorizado (OCA), un auditor de cuentas registrado o la propia Administración.

\subsection{Ayudas de financiación indirecta}

Las ayudas de financiación indirectas a las actividades de I+D+i suponen, en esencia, una fiscalidad especial resultante de la aplicación de deducciones a la cuota del impuesto de sociedades (IS).

En este caso, los incentivos fiscales están enfocados al impulso de la investigación, el desarrollo y la innovación. Frente a otros instrumentos de fomento de la $\mathrm{I}+\mathrm{D}+\mathrm{i}$, las deducciones fiscales presentan tres ventajas principales: a) efectos económicos equiparables a los de una subvención, pero están exentos de tributación; b) son de aplicación libre y general al no estar sometidas a concurrencia competitiva con un presupuesto predefinido; y c) son proporcionales a las actividades de I+D+i desarrolladas, cabiendo la posibilidad de ser aplicadas en ejercicios posteriores.

Las deducciones fiscales en el impuesto de sociedades son básicamente de dos tipos: deducciones por I+D o innovación tecnológica y cesión de activos intangibles o Patent Box.

\subsubsection{Deducciones fiscales}

\subsubsection{Procedimiento y gestión}

La principal referencia para la aplicación de estas deducciones por actividades de investigación y desarrollo e innovación tecnológica la constituye el artículo 35 de la Ley 27/2014 del Impuesto sobre Sociedades.

Las deducciones fiscales se practican sobre la cuota íntegra ajustada del impuesto. Si la cuota íntegra es positiva, la deducción es de aplicación inmediata. Si la cuota íntegra resultara ser negativa, dará lugar a dos posibilidades: la generación de la deducción (es decir, devengar el derecho a deducirse en el futuro) 0 bien la posibilidad de solicitar el abono por parte de la Administración, a partir de la promulgación de la Ley 14/2013 de Apoyo a los Emprendedores y su Internacionalización.

El límite de las deducciones fiscales se establece en el $50 \%$ de la cuota íntegra del impuesto sobre sociedades, ampliado posteriormente al $60 \%$ a partir de los ejercicios fiscales iniciados a partir del 6 de marzo de 2011, de acuerdo con la Ley de Economía Sostenible.

En las deducciones por innovación, el procedimiento para poder obtener la calificación favorable esta reglado y, por tanto, no debe de comportar excesivos problemas. En cambio, cuando se trata de deducciones por investigación y desarrollo, el proceso que conlleva para que sea aprobado es más complejo.

En cualquier caso, para acceder a estas deducciones fiscales a la I+D+i, la empresa está obligada a estructurar sus gastos de I+D+i en proyectos. Además, para poder aplicar deducciones por $\mathrm{I}+\mathrm{D}+\mathrm{i}$, se tiene que calificar la naturaleza de las actividades de los proyectos siguiendo la definición fiscal de investigación, desarrollo e innovación tecnológica que establece la propia legislación.

\subsubsection{Calificación de proyectos}

Esta calificación puede realizarse por dos vías principales: vía tradicional o directa y vía informe motivado. 
En la vía tradicional, la empresa puede solicitar la desgravación fiscal directamente a la Agencia Tributaria, sin necesidad de que un tercero acredite previamente que las actividades deducibles constituyen I+D+i. Esta vía tiene ciertos riesgos, como la dificultad para identificar los distintos tipos de actividades de I+D+i, así como para imputar correctamente los gastos correspondientes.

La vía del informe motivado es un mecanismo que pretende dar a la empresa solicitante la seguridad jurídica de que la Agencia Tributaria no cuestionará la naturaleza de I+D o innovación tecnológica del proyecto que cuenta con dicho informe.

El informe motivado es un documento emitido por determinados organismos oficiales $y$ que se presenta a la Agencia Tributaria como acreditación de la naturaleza de las actividades de I+D+i a deducir.

Son dos los organismos habilitados por ley para emitir informes motivados vinculantes: el Ministerio de Industria, Comercio y Turismo, y el CDTI.

El Ministerio de Industria, Comercio y Turismo puede emitir un informe motivado ex post para cada ejercicio fiscal del proyecto ejecutado y cerrado. Este informe previamente debe ser validado a través de alguna de las entidades acreditadas por la Entidad Nacional de Acreditación (ENAC). Una vez validado por ENAC, la empresa solicitante aportará al ministerio, telemáticamente, la solicitud de informe motivado junto con el entregable para solicitud de informe motivado emitido por ENAC.

El CDTI es un órgano competente para emitir informes vinculantes en los proyectos que financia ${ }^{4}$.

\footnotetext{
4 Para que el CDTI pueda emitir informe motivado es necesario que se haya presentado un proyecto de $I+D+i$ y que haya sido financiado $y$ subvencionado por parte del órgano, como paso previo para que se pueda emitir el informe motivado.
}

En la emisión del informe motivado vinculante por parte del CDTI se certifica el contenido ex ante en actividades de $\mathrm{I}+\mathrm{D}+\mathrm{i}$, tal como se define en Ley 27/2014 del Impuesto sobre Sociedades, así como la coherencia de los gastos presupuestados para la realización de las actividades de I+D+i contempladas en el mismo.

Para solicitar el informe motivado vinculante al CDTI, previamente este organismo debe haber calificado el proyecto como de I+D+i. Una vez calificado como tal, el CDTI concederá una financiación y una subvención aplicada a reducir el principal del crédito. Una vez emitido el informe favorable que certifica el contenido ex ante, la empresa podrá solicitar, telemáticamente, la emisión del informe motivado vinculante al CDTI.

\subsubsection{La ineficacia de los incentivos fiscales en España}

A raíz de la grave crisis internacional de 2007, la mayoría de las empresas españolas que solicitaban las deducciones fiscales, a través de los informes motivados, iban acumulando bonificaciones fiscales en la medida que no generaban beneficios suficientes para poder aplicarlas.

Con la promulgación de la Ley 14/2013 del emprendedor, y mediante el artículo 26 , se pretendió corregir esta dificultad al establecerse que, en caso de insuficiencia de la cuota íntegra, las pymes podrían solicitar el abono a la Administración tributaria de la deducción por anticipado, renunciando a un $20 \%$ del importe de la deducción aprobada. Con esta medida, a priori, se ofrecían nuevas posibilidades a las empresas para que impulsasen proyectos de I+D+i, sin que la causa de una cuota íntegra insuficiente fuese un obstáculo en la aplicación de deducciones fiscales. Con todo, las $\square$ 
bondades de esta medida quedaron rápidamente neutralizadas por una cuestión procedimental. Solo podían beneficiarse del abono aquellas empresas que hubieran solicitado un informe motivado al Ministerio de Industria, Comercio y Turismo. Esto significaba que el informe motivado emitido por el CDTI no aplicaba en la solicitud de abono de la deducción.

Este extremo ha provocado que el abono de la deducción no haya resultado una medida tan efectiva como inicialmente se preveía, hasta el punto que prácticamente ninguna empresa ha solicitado el abono de la deducción por la inseguridad jurídica que se crea frente a la Administración tributaria.

\subsubsection{Patent Box o cesión de activos intangibles}

\subsubsection{Definición y esquema de funcionamiento}

El Patent Box es una figura legal basada en la cesión de uso de activos intangibles que persigue incentivar, mediante una reducción del $60 \%$ en la base imponible del impuesto de sociedades, la generación de rentas positivas derivadas de la explotación de activos intangibles como patentes, dibujos, modelos, fórmulas, planos o procedimientos, con la finalidad de impulsar su desarrollo e internacionalización.

El Patent Box tiene su origen en el entorno fiscal europeo y la Directiva Comunitaria Interest \& Royalty (2003/49/C) y con efectos en España desde el periodo impositivo de enero de 2008. La Ley 14/2013 de Apoyo a los Emprendedores y su Internacionalización introdujo mejoras significativas en este régimen fiscal para aquellas cesiones de intangibles realizadas a partir de septiembre de 2013 , con la finalidad de impulsar el desarrollo e internacionalización de activos empresariales intangibles. Actualmente, el Patent Box se encuentra implantado en doce países dentro del ámbito europeo, que son: Bélgica, Chipre, España, Francia, Países Bajos, Hungría, Italia, Luxemburgo, Malta, Portugal, Reino Unido e Irlanda.

\subsubsection{Esquema de funcionamiento}

El esquema de funcionamiento del Patent Box, ilustrado en la Figura 1, contempla a dos actores: 1) una empresa cedente, generadora del conocimiento y propietaria del activo intangible objeto de la cesión, normalmente registrada bajo una patente; y 2) una empresa $\triangle$

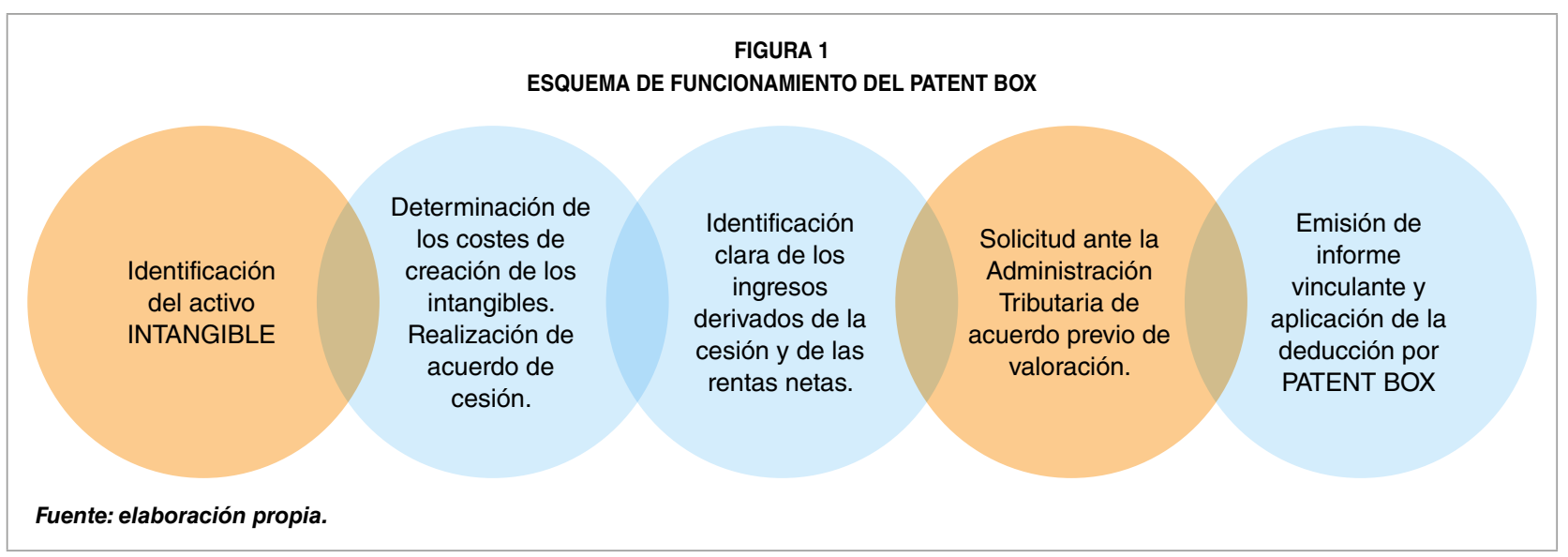


cesionaria que utiliza el activo intangible (que ha recibido en cesión) para desarrollar su actividad económica.

En esencia, la lógica del Patent Box consiste en proporcionar un beneficio fiscal a la empresa generadora del conocimiento y propietaria del activo intangible cuando esta transmite su conocimiento, vía cesión de un activo intangible, a otra empresa, que emplea ese activo para desarrollar su actividad.

En consecuencia, este régimen fiscal, al reducir la base imponible de la empresa cesionaria del activo intangible, proporciona un incentivo a la innovación y desarrollo empresarial.

\subsubsection{Requisitos legales}

La posibilidad de beneficiarse de las ventajas fiscales que supone el esquema del $\mathrm{Pa}-$ tent Box está sujeta al cumplimiento de una serie de requisitos. Así, la cesión de los activos intangibles solo es posible si la entidad cedente ha creado los activos objeto de la cesión; esto significa, en la práctica, que la empresa propietaria de la patente puede acreditar que ha soportado al menos un $25 \%$ de su coste.

La empresa cesionaria que explota económicamente el activo intangible debe ser una compañía no residente en paraíso fiscal, excepto que esté situada en un Estado miembro de la UE y el sujeto pasivo acredite que la operativa responde a motivos económicos válidos. Además, el cesionario debe utilizar los derechos de uso del activo intangible en el desarrollo de una actividad económica determinada. En el caso de que la empresa cedente y la empresa cesionaria estén vinculadas, los resultados derivados de la explotación de la patente no pueden materializarse en la entrega de bienes o prestaciones de servicios por parte del cesionario que generen gastos fiscalmente deducibles en la entidad cedente.

También se requiere que la empresa cedente y la empresa cesionaria dispongan de registros contables diferenciados, necesarios para poder determinar los ingresos y gastos, directos e indirectos, correspondientes a los activos objeto de cesión. En este sentido, cuando un mismo contrato de cesión incluya prestaciones accesorias de servicios, debe diferenciarse en dicho contrato la contraprestación correspondiente a los mismos.

El régimen fiscal del Patent Box permite que la tributación recaiga sobre las rentas netas generadas por la cesión de activos intangibles. En este sentido, la renta neta es la diferencia positiva entre los ingresos del ejercicio procedentes de la cesión del derecho de uso y las cantidades que sean deducidas en el mismo por deterioro y gastos relacionados con el intangible. Si los activos no están reconocidos en balance, se entenderá como renta neta generada por el activo intangible el $80 \%$ de los ingresos procedentes de la cesión.

\subsubsection{Casuística del Patent Box: un análisis comparativo}

Con el ánimo de ilustrar el funcionamiento del Patent Box y mostrar cómo los beneficios fiscales que se derivan pueden constituir un incentivo real a la innovación empresarial planteamos el caso de una sociedad $A$ que ha desarrollado una patente cuyo coste se cifra en 150.000 euros, amortizable anualmente durante cinco años a razón de 30.000 euros anuales. La empresa $A$ se plantea la alternativa de explotar esa patente mediante un esquema $\mathrm{Pa}-$ tent Box o hacerlo directamente sin ceder el uso de la misma. 
Caso 1. Esquema modelo de cesión con Patent Box

La sociedad A, con la finalidad de obtener un rendimiento de la patente desarrollada y generar un beneficio fiscal, decide desarrollar un esquema Patent Box para un periodo de cinco años.

Para ello, la sociedad A cede temporalmente a la sociedad $B$ la patente o activo intangible.

El contrato de cesión de uso se articula a través de una tarifa en dos partes: una parte fija de 40.000 euros anuales, más una parte variable a razón de 15 euros por unidad vendida.

La sociedad B está interesada en esta cesión temporal porque la incorporación de la patente a su actividad ha de permitirle desarrollar una nueva línea de negocio que aumentará sus ingresos por ventas.

En esta lógica, la sociedad A (cedente) obtiene unos ingresos acumulados de 1.287 .500 euros por la cesión del activo intangible a la sociedad B (cesionaria), durante los cinco años de duración del contrato. Suponemos que en este periodo de tiempo el número de unidades vendidas en promedio ha sido de 14.500 , lo cual determina un total de 72.500 unidades para el conjunto del periodo. En virtud del contrato de cesión de uso, a la sociedad A le corresponde un ingreso fijo de 200.000 euros anuales, a razón de 40.000 euros/año, más 1.087 .500 euros de ingreso total acumulado por la parte variable de la tarifa ${ }^{5}$. Adicionalmente, la sociedad A (cedente) incurre en unos gastos de amortización del activo intangible que suman 150.000 euros del periodo de cinco años.

5 Ingresos acumulados por parte variable = precio de venta $\mathrm{x}$ número de unidades vendidas $=15 € / \mathrm{u} \times 72.500 \mathrm{u}=1.087 .500 €$.
Por otra parte, la sociedad B (cesionaria), a partir del uso de la patente que le ha sido cedida, obtiene unos ingresos de 1.612 .500 euros acumulados a lo largo de los cinco años. Así mismo, esta empresa también asume como gasto propio el importe de los derechos de uso de la patente (1.287.500 euros) que ha pagado a la sociedad A (cedente) durante el periodo de vigencia del contrato.

Con esta información, la renta neta de la sociedad A (cedente) se estima en 1.137 .500 euros, una cuantía que resulta de la diferencia entre los ingresos brutos (1.287.500 euros) percibidos por el derecho de uso de la patente en este ejercicio por parte de la sociedad B (cedente) y los gastos de la amortización acumulada de la patente (150.000 euros).

Por su parte, la renta neta de la sociedad B (cesionaria) asciende a 325.000 euros. Este importe resulta de la diferencia entre los ingresos por ventas (1.612.500 euros) de la línea de negocio de la sociedad B (cesionaria) en la que interviene la patente cedida y los gastos (1.287.500 euros) por el derecho de uso de la patente y pagados a la sociedad $A$ (cedente).

En la medida que esta colaboración entre la sociedad A (cedente) y la sociedad B (cesionaria) se articula con base en un esquema de $\mathrm{Pa}$ tent Box, fiscalmente el resultado sería el que se describe a continuación y se muestra en la Tabla 1.

En virtud del régimen fiscal del Patent Box, el $60 \%$ de la renta neta de la sociedad A (cedente) quedaría exenta de tributación (682.500 euros). Esta exención recibe el nombre de «Ajuste Patent Box». En consecuencia, la base imponible del impuesto de sociedades para esta sociedad A (cedente) sería igual a 455.000 euros, equivalentes al $40 \%$ de la renta neta generada. De ahí que, en términos del $\triangleright$ 
TABLA 1

MODELO CON PATENT BOX

\begin{tabular}{|c|c|c|c|c|c|}
\hline \multicolumn{2}{|l|}{ Sociedad cedente (A) } & & \multicolumn{2}{|c|}{ Sociedad cesionaria (B) } & Total impuesto a pagar \\
\hline \multicolumn{2}{|c|}{ Generadora del conocimiento } & & \multicolumn{2}{|c|}{ Usa el activo en una actividad económica } & \\
\hline & & A & 1. Ingresos por ventas & 1.612 .500 & \\
\hline 1. Ingresos por cesión & 1.287 .500 & $\mathrm{~B}$ & 2. Gastos cesión intangible & 1.287 .500 & \\
\hline 2. Gastos I+D (amortización) & 150.000 & & & & \\
\hline 3. Renta neta (1-2) & 1.137 .500 & & 3. Renta neta (1-2) & 325.000 & \\
\hline Ajuste Patent Box (60\% renta neta) & 682.500 & & & & \\
\hline Base imponible IS & 455.000 & & Base imponible IS & 325.000 & \\
\hline Impuesto de sociedades (25\%) & 113.750 & & Impuesto de sociedades (25\%) & 81.250 & 195.000 \\
\hline
\end{tabular}

esquema Patent Box, la base imponible equivale a la renta neta de la sociedad cedente menos el ajuste Patent Box. Si sobre esta base imponible ajustada por el Patent Box suponemos que se aplica un tipo impositivo del $25 \%$, entonces la cuota líquida a pagar por parte de la sociedad A (cedente) sería de 113.750 euros, en términos acumulados a lo largo de los cinco años.

Por otra parte, la base imponible de la sociedad B (cesionaria) se estima en 325.000 euros, una cuantía equivalente a la renta neta de esta empresa. Si sobre esta base imponible suponemos que se aplica un tipo impositivo del $25 \%$, entonces la cuota líquida a pagar por parte de la sociedad B (cesionaria) sería de 81.250 euros para los cinco años considerados.

La recaudación total en el conjunto del periodo de la Agencia Tributaria ascendería a 195.000 euros, resultado de agregar las cuotas líquidas ingresadas por la sociedad $A$ (cedente), de 113.750 euros, y por la sociedad B (cesionaria), de 81.250 euros.

\section{Caso 2. Esquema modelo sin Patent Box}

Para poder valorar adecuadamente el alcance y la trascendencia del esquema Patent
Box, formulamos ahora un segundo caso de estudio, en el que suponemos que no se produce la cesión de activos intangibles. Ahora, por homogeneidad en el cálculo, suponemos que la sociedad A obtiene los mismos ingresos por ventas a los imputados en el caso anterior a la sociedad B, para el conjunto del periodo de cinco años considerados. Estos ingresos ascienden a 1.612 .500 euros, de los cuales deben descontarse los gastos de amortización acumulados de la inversión en $\mathrm{I}+\mathrm{D}+\mathrm{i}$ y que ascienden a 150.000 euros.

En consecuencia, ahora la renta neta de la sociedad A será de 1.462 .500 euros. Esta renta neta determina la base imponible de esta empresa. Si sobre esta base imponible suponemos que se aplica un tipo impositivo del $25 \%$, entonces la cuota líquida a pagar acumulada durante los cinco años por parte de la sociedad A sería de 365.625 euros. Este importe equivale también a la recaudación total de la Agencia Tributaria por este motivo, tal como se recoge en la Tabla 2. En consecuencia, el esquema Patent Box supone un ahorro fiscal evidente respecto a aquella situación en la que no utiliza el Patent Box. En los casos que hemos ejemplificado, este ahorro fiscal se ha cifrado en 176.625 euros para los cinco años de amortización del activo intangible. 
TABLA 2

MODELO SIN PATENT BOX

\begin{tabular}{|l|c|c|}
\hline \multicolumn{2}{|c|}{ Sociedad A (propietaria) } & Total impuesto a pagar \\
\hline Generadora y comercializadora del activo, no hay cesión & 1.612 .500 & \\
Ingresos por ventas & 150.000 & \\
Gastos I+D (amortización) & 1.462 .500 & \\
Renta neta (A-B) & 1.462 .500 & 365.625 \\
Base imponible IS & 365.625 \\
\hline Impuesto de sociedades (25\%) & \\
\hline Fuente: elaboración propia.
\end{tabular}

\section{Conclusiones}

El indicador de innovación en Europa de la Comisión Europea 2020, que valora las fortalezas y debilidades de cada país en términos de innovación, sitúa la economía española entre los países con un desempeño en innovación por debajo de la media europea, aunque su posición relativa ha mejorado entre 2012 y 2019. Las principales diferencias se concentran en la inversión en innovación realizada por el sector empresarial. En términos comparados, el esfuerzo inversor $(92,6)$ en España es moderado y representa el $85 \%$ de la media de la UE-27, un registro todavía muy alejado de los valores de países líderes en innovación como Suiza $(179,7)$, Suecia $(153,1)$, Finlandia $(152,2)$ o Dinamarca $(146,4)$, o países con una potente capacidad de innovación como Reino Unido $(131,6)$, Alemania $(130,5)$ o Irlanda $(121,9)$. Esta menor intensidad de la innovación en el caso de España se explica fundamentalmente a partir de la menor inversión empresarial en innovación, imputable a la estructura sectorial de la economía, a un tejido empresarial configurado esencialmente por pymes, pero también a una cultura industrial con poca tradición de colaboración y transferencia tecnológica entre empresas y centros de investigación público y privados, al escaso desarrollo del capital riesgo o al propio diseño de la políticas de incentivos de I+D+i.
En este contexto, el esquema Patent Box se erige en un incentivo fiscal efectivo en la promoción de proyectos de I+D+i y la atracción de inversiones internacionales, a diferencia de las deducciones fiscales cuyo procedimiento administrativo proceloso y la fijación de un descuento máximo sobre la cuota íntegra del impuesto de sociedades, que se ha revelado como insuficiente, han tendido a minar su protagonismo como instrumento de ayuda indirecta a la I+D+i.

\section{Bibliografía}

Comisión Europea (2014). Marco sobre Ayudas Estatales de Investigación y Desarrollo e Innovación. 2014/C 198/01. Comunicación de la Comisión. Diario Oficial de la Unión Europea. https:// eur-lex.europa.eu/legal-content/ES/TXT/ PDF/?uri=CELEX:52014XC0627(01)\&from=EL

Comisión Europea (2018). Informe Nacional RIO 2017: Spain. Research and Innovation Observatory (RIO). JRC Science for Policy Report.

Comisión Europea (2019). European Innovation Scoreboard 2019. https://ec.europa.eu/growth/ industry/innovation/facts-figures/scoreboards en

Ley 2/2011, de 4 de marzo, de Economía Sostenible. Jefatura del Estado. Boletín Oficial del Estado, n. -55 , de 5 de marzo de 2011. Referencia: BOE-A-2011-4117. 
Ley 14/2013, de 27 de septiembre, de Apoyo a los Emprendedores y su Internacionalización. Jefatura del Estado. Boletín Oficial del Estado, n. -233 , de 28 de septiembre de 2013. Referencia: BOE-A-2013-10074.

Ley 27/2014, de 27 de noviembre, del Impuesto sobre Sociedades. Jefatura del Estado. Boletín Oficial del Estado, n.․ 288, de 28 de noviembre de 2014. Referencia: BOE-A-2014-12328.

Lucas, R. (1998). On the Mechanics of Economic Development. Journal of Monetary Economics, 22 (July), 3-42.

OCDE / EUROSTAT (2018). Oslo Manual 2018: Guidelines for collecting, reporting and using data on innovation, $4^{\text {th }}$ Edition. The Measurement of Scientific, Technological and Innovation Activities. Organización para la Cooperación y el Desarrollo Económico (OCDE) y la Oficina de Estadística de la Unión Europea (Eurostat) y CDE referencia en temas de innovación). OECD Publishing. https:// doi.org/10.1787/9789264304604-en

Romer, P. (1994). The origins of endogenous growth. The Journal of Economic Perspectives, 8 (1), 3-22.

Solow, R. (1956). A contribution to the theory of economic growth. The Quarterly Journal of Economics, 70 (1), 65-94. 